\title{
Structural brain changes associated with tardive dyskinesia in schizophrenia ${ }^{\dagger}$
}

Salvador Sarró, Edith Pomarol-Clotet, Erick J. Canales-Rodríguez, Raymond Salvador, Jesús J. Gomar, Jordi Ortiz-Gil, Ramón Landín-Romero, Fidel Vila-Rodríguez, Josep Blanch and Peter J. McKenna

\section{Background}

The pathological basis of tardive dyskinesia is unknown. Although its clinical features implicate the basal ganglia, imaging studies have not found clear evidence that it is associated with volume changes in these or other brain structures.

\section{Aims}

To determine, using voxel-based structural imaging, whether there are regions of grey matter volume change in people with schizophrenia who also have tardive dyskinesia compared with those without tardive dyskinesia.

\section{Method}

A total of 81 people with chronic schizophrenia, 32 with tardive dyskinesia and 49 without, were examined using magnetic resonance imaging (MRI) and whole-brain, optimised voxel-based morphometry. A comparison group of 61 healthy controls was also examined.

\section{Results}

Compared with those without tardive dyskinesia, patients with tardive dyskinesia showed a pattern of volume reductions in predominantly subcortical regions, including the basal ganglia and the thalamus. Within the basal ganglia, volume reductions were seen in the caudate nucleus, to a lesser extent in the putamen, and only marginally in the globus pallidus. The patients with tardive dyskinesia, but not those without, showed significant volume reductions in the basal ganglia compared with the healthy controls but both groups had smaller volumes than controls in other affected areas.

\section{Conclusions}

The pathological process or processes that underlie the development of tardive dyskinesia are not just neurochemical in nature, but affect brain structure.

\section{Declaration of interest}

None.
Tardive dyskinesia is a syndrome of involuntary movements affecting the face and/or the trunk and extremities, which is seen in $20-25 \%$ of patients with schizophrenia. ${ }^{1}$ Once it appears it commonly becomes irreversible, and it may sometimes be disabling or in rare cases fatal. ${ }^{1}$ It typically occurs in patients who have received months or years of antipsychotic treatment. As a result it is classified as a late-developing extrapyramidal side-effect of this class of drugs.

Considering tardive dyskinesia simply as a side-effect of antipsychotics may, however, not be entirely justified. This is because essentially similar involuntary movements can also be seen in people with schizophrenia who have not received treatment. According to current estimates, such spontaneous dyskinesias are present in around $4 \%$ of patients with first-episode schizophrenia ${ }^{2}$ and the frequency may reach $40 \%$ in the (nowadays rare) populations of people with chronic schizophrenia who have never been exposed to antipsychotics. ${ }^{3,4}$ To account for this finding, it has been proposed that there is an intrinsic vulnerability to involuntary movements in schizophrenia and the role of antipsychotics is one of promotion or acceleration rather than causation. ${ }^{1,5}$ More radically, Crow et al ${ }^{6}$ have argued that, along with negative symptoms and cognitive impairment, tardive dyskinesia is part of the pattern of deterioration associated with the illness and antipsychotic treatment plays little or no part in its emergence.

Despite its clinical importance, little is known about the biological basis of tardive dyskinesia. The major hypothesis over the years has been that it reflects an increase in dopamine $\mathrm{D}_{2}$ receptor numbers in the basal ganglia. ${ }^{7}$ This view was originally based on evidence implicating a functional dopamine excess in choreoathetoid movement disorders across a range of neurological

'See editorial, pp. 6-7, this issue. disease states, ${ }^{8}$ and on observations that tardive dyskinesia can be suppressed and exacerbated by dopamine antagonist and agonist drugs respectively. ${ }^{9}$ However, post-mortem ${ }^{10-12}$ and in vivo studies using radioligand imaging (for references see Alder et $a l^{13}$ ) have uniformly failed to demonstrate differences in dopamine $\mathrm{D}_{2}$ receptor numbers in people with schizophrenia with and without tardive dyskinesia.

Another avenue of research in tardive dyskinesia has been to try and determine whether it is associated with structural brain changes. An early computed tomography (CT) study found that individuals with schizophrenia and tardive dyskinesia had larger lateral ventricles than those without, ${ }^{14}$ but further studies failed to confirm this finding. ${ }^{15}$ Using MRI, Mion et al ${ }^{16}$ found a smaller volume of the caudate nucleus, but not other basal ganglia nuclei, in people with schizophrenia with tardive dyskinesia compared with those without. However, this finding has again not been consistently replicated in subsequent studies. ${ }^{17,18}$ There are a number of obstacles to detecting brain structural differences between patients with and without tardive dyskinesia. One is that the changes in schizophrenia in comparison to the healthy population are small - for example, the overall reduction of brain volume is about $2 \% .{ }^{19}$ Another is that treatment with antipsychotic drugs has been found to cause volume increases in the basal ganglia, ${ }^{20}$ which could obscure volume reductions associated with tardive dyskinesia in this region. A final problem is that conventional brain imaging requires the selection of predetermined regions for study, which in the case of tardive dyskinesia has led to a focus on the basal ganglia; other components of the extrapyramidal system, such as the thalamus, substantia nigra, premotor cortex and prefrontal cortex, have not been investigated.

The development of structural imaging methods that map clusters of significant difference throughout the brain between 
groups of participants, without the necessity of a priori selection of regions of interest, has obvious advantages for answering questions about brain changes associated with tardive dyskinesia. In this study we applied one such technique, voxel-based morphometry (VBM), to a group of people with schizophrenia with and without tardive dyskinesia. We also included a healthy control group in order to be able to determine the relationship of any changes found to the brain volume changes associated with schizophrenia itself.

\section{Method}

\section{Participants}

The patient sample consisted of 81 adult in- and out-patients with schizophrenia (age range 23-63) drawn from two psychiatric hospitals, Benito Menni CASM and Sant Joan de Déu SSM in Barcelona, Spain. The sample was made up of two subgroups of patients selected on the basis of whether they showed $(n=32)$ or did not show $(n=49)$ tardive dyskinesia. All patients met ${ }^{D S M}-\mathrm{IV}^{21}$ criteria for schizophrenia, based on interview by two psychiatrists plus review of case notes. Patients were excluded if: (a) they were younger than 18 or older than 65 years; (b) they had a history of brain trauma or neurological disease; and (c) they had shown alcohol/substance misuse within the 12 months prior to participation. Patients also needed to have a current IQ in the normal range (i.e. 70+). All patients were right handed. They were all on treatment with antipsychotics: atypical $(n=46)$, typical $(n=7)$, combined typical and atypical treatment $(n=27)$ (detailed drug information was missing for one patient).

A group of 61 healthy controls was also employed. They were selected to be demographically matched to the whole group of patients, and to the patients with and without tardive dyskinesia. They met the same exclusion criteria as the patients. They were recruited from non-medical staff working in the hospital, their relatives and acquaintances, and also from independent sources in the community. They were questioned and excluded if they reported a history of mental illness and/or treatment with psychotropic medication. The study was approved by the local research ethics committee and all patients gave written informed consent after a detailed explanation of the study.

\section{Evaluation of motor disorder}

Motor disorder was rated in the patients using a standard examination, which was videotaped. Patients were examined while seated, standing and walking, and 'activation' procedures designed to elicit involuntary movements were employed (touching the thumb of each hand to each finger in turn, holding the arms out with the wrists flexed, reciting the months of the year backwards). Ratings on Simpson et al's ${ }^{22}$ scale for tardive dyskinesia were made by two raters together (S.S. and E.P.-C.) who had been trained in the assessment of extrapyramidal sideeffects. Videos of all patients who showed any evidence of involuntary movements were then reviewed by a third rater (P.J.M.) who had extensive experience in rating tardive dyskinesia; final ratings were made by consensus.

Presence of tardive dyskinesia was defined according to Schooler \& Kane's criteria. ${ }^{23}$ These require moderate involuntary movements (rating of $\geqslant 3$ on the Simpson scale) in at least one body area, or mild involuntary movements (rating of 2) in at least two different body areas. We additionally required that the patients show positive ratings on the core dyskinetic items on the Simpson scale; any who only scored on the items: increased blinking, tremor of eyelids, tremor of upper lip, tongue tremor, caressing/rubbing face, hair or thighs, restless legs, crossing/ uncrossing legs or akathisia were not considered as having tardive dyskinesia. Patients without tardive dyskinesia scored no more than 1 (questionable) on any of the dyskinesia items on the Simpson scale.

\section{Other measures}

Symptoms were scored using the Spanish version of the Positive and Negative Syndrome Scale (PANSS). ${ }^{24}$ Premorbid IQ was estimated using the Word Accentuation Test (Test de Acentuación de Palabras, TAP), ${ }^{25}$ a word reading test that requires pronunciation of Spanish words whose accents have been removed. Current IQ was prorated from four subtests of the Wechsler Adult Intelligence Scale III (WAIS-III) ${ }^{26}$ (vocabulary, similarities, block design, and matrix reasoning).

\section{MRI data acquisition}

All participants underwent structural MRI scanning in the same 1.5 Tesla GE Signa scanner (General Electric Medical Systems, Milwaukee, Wisconsin, USA) located at the Sant Joan de Déu Hospital in Barcelona (Spain). High resolution structural $T_{1}$ MRI data were acquired with the following acquisition parameters: matrix size $512 \times 512 ; 180$ contiguous axial slices; voxel resolution $0.47 \times 0.471 \mathrm{~mm}^{3}$; echo $(\mathrm{TE})$, repetition $(\mathrm{TR})$ and inversion (TI) times $3.93 \mathrm{~ms}, 2000 \mathrm{~ms}$ and $710 \mathrm{~ms}$ respectively; flip angle $15^{\circ}$.

Structural data were analysed with FSL-VBM, an optimised voxel-based morphometry style analysis ${ }^{27}$ carried out with FSL tools; ${ }^{28}$ this yields a measure of difference in local grey matter volume. In a first step, structural images were brain-extracted using BET. ${ }^{29}$ Next, tissue-type segmentation was carried out and the resulting grey matter partial volume images were then aligned to Montreal Neurological Institute (MNI 152) standard space using the FSL tools FLIRT and FNIRT. The resulting images were averaged to create a study-specific template, to which the native grey matter images were then non-linearly re-registered. The registered partial volume images were then modulated (to correct for local expansion or contraction) by dividing by the Jacobian of the warp field. The modulated segmented images were then smoothed with an isotropic Gaussian kernel with a sigma of $4 \mathrm{~mm}$.

\section{Data analysis}

Group comparisons were carried out using a voxel-wise general linear model (GLM) and permutation-based non-parametric testing (for more technical details see www.fmrib.ox.ac.uk/fsl/ fslvbm/index.html), correcting for multiple comparisons. These were made with the randomise programme implemented in FSL, using a cluster-based thresholding method with 10000 iterations and initial cluster-forming threshold $Z \geqslant 2.3$. The GLM was designed to account for the gender-related variability between participants. Clusters were assessed for significance at $P<0.05$, fully corrected for multiple comparisons across space. Anatomical locations of the significant clusters were determined by reference to the Harvard-Oxford cortical structural atlas integrated into FSL view (part of FSL) and the AAL atlas of 116 segmented structures within MRIcron software (for more details see www. mccauslandcenter.sc.edu/mricro/mricron/index.html).

\section{Results}

\section{Demographic features of the patients and controls}

The findings are shown in Table 1. The controls were well matched to the whole group of patients (age: $t=-1.34, P=0.18$; gender: 
Table 1 Demographic and clinical data on the patients

\begin{tabular}{|c|c|c|c|c|}
\hline & \multirow[b]{2}{*}{$\begin{array}{l}\text { Controls } \\
(n=61)\end{array}$} & \multicolumn{2}{|c|}{ Participants with schizophrenia $(n=81)$} & \multirow[b]{2}{*}{$P$} \\
\hline & & $\begin{array}{l}\text { Tardive dyskinesia } \\
\text { group }(n=32)\end{array}$ & $\begin{array}{l}\text { Non-tardive dyskinesia } \\
\text { group }(n=49)\end{array}$ & \\
\hline Age, years: mean (s.d.) & $40.69(10.11)$ & $43.09(10.27)$ & $42.82(9.43)$ & 0.41 \\
\hline Female/male, $n$ & $17 / 44$ & $7 / 25$ & $15 / 34$ & 0.69 \\
\hline Estimated premorbid IQ, TAP: mean (s.d.) & $100.63(18.59)$ & $95.74(12.5)$ & $98.89(9.86)$ & 0.15 \\
\hline Current IQ, Wechsler Adult Intelligence Scale III: mean (s.d.) & $104.60(18.59)$ & $93.44(12.45)$ & $92.92(10.73)$ & $<0.001^{a}$ \\
\hline Illness duration, years: mean (s.d.) & & $22.36(10.89)$ & $20.81(8.00)$ & 0.52 \\
\hline Positive and Negative Syndrome Scale, total score: mean (s.d.) & & $76.07(16.04)$ & $73.57(15.84)$ & 0.73 \\
\hline Positive syndrome & & $14.45(5.08)$ & $16.00(5.30)$ & 0.21 \\
\hline Negative syndrome & & $17.10(6.34)$ & $16.94(5.25)$ & 0.90 \\
\hline Disorganisation syndrome & & $10.86(4.10)$ & $9.14(2.87)$ & 0.03 \\
\hline Global Assessment of Functioning Scale score, mean (s.d.) & & $41.70(11.71)$ & $41.98(10.69)$ & 0.92 \\
\hline Tardive dyskinesia score, mean (s.d.) & & $10.13(7.92)$ & $0.51(1.00)$ & 0.00 \\
\hline \multicolumn{5}{|l|}{ Current antipsychotic treatment, ${ }^{\mathrm{b}} \mathrm{n}(\%)$} \\
\hline Typical & & $2(6.3)$ & $5(10.2)$ & 0.54 \\
\hline Atypical & & $19(56.3)$ & $27(55.1)$ & 0.92 \\
\hline Typical and atypical & & $10(34.4)$ & $17(34.7)$ & 0.98 \\
\hline Antipsychotic dose in chlorpromazine equivalents, mg: $^{\cdot b}$ mean (s.d.) & & $1035.07(761.16)$ & $791.18(440.16)$ & 0.14 \\
\hline Duration of treatment, ${ }^{\mathrm{b}} \mathrm{n}$ & & & & $0.37^{\mathrm{C}}$ \\
\hline$<1$ year & & 0 & 2 & \\
\hline $1-5$ years & & 0 & 1 & \\
\hline$>5$ years & & 26 & 38 & \\
\hline Uncertain & & 5 & 8 & \\
\hline $\begin{array}{l}\text { TAP, Word Accentuation Test (Test de Acentuación de Palabras). } \\
\text { a. Controls > tardive dyskinesia group; controls }>\text { non-tardive dyskinesia group. } \\
\text { b. Missing data for one participant in the tardive dyskinesia group. } \\
\text { c. Analysis carried out excluding participants in 'uncertain' category. }\end{array}$ & & & & \\
\hline
\end{tabular}

$\chi^{2}=0.01, P=0.92$; estimated premorbid IQ: $\left.t=1.52, P=0.13\right)$ and to the patients with and without tardive dyskinesia. The tardive dyskinesia and non-tardive dyskinesia groups were similar in age, but differed in gender distribution ( $22 \%$ v. 31\% female), although not significantly. Since frequency of tardive dyskinesia has been found to vary according to gender in some studies, ${ }^{1}$ this minor gender difference was covaried for in the comparison between patients with and without tardive dyskinesia. There were no significant differences between the two patient groups in duration of illness, global severity of illness or antipsychotic dosage in chlorpromazine equivalents (although this was numerically higher in the patients with tardive dyskinesia). They also showed similar levels of overall symptomatology as measured using the PANSS; however, the patients with tardive dyskinesia had significantly higher scores on the PANSS disorganisation factor compared with those without.

Length of antipsychotic treatment in the patients with and without tardive dyskinesia is also shown in Table 1. Most patients had received treatment with both typical and atypical antipsychotics; 1 patient (without tardive dyskinesia) had been treated only with typical antipsychotics, and 5 (all without tardive dyskinesia) had been treated only with atypicals. Most patients had received more than 5 years of antipsychotic treatment; this applied both to conventional antipsychotics (with tardive dyskinesia $80.8 \%$, without tardive dyskinesia $65.9 \%$ ) and atypicals (with tardive dyskinesia 79.1\%, without tardive dyskinesia $80.8 \%$ ).

Of the patients with tardive dyskinesia, 7 showed only orofacial movements (based on a score of $\geqslant 2$ in any facial region), 10 showed only trunk and/or limb movements (based on a score of $\geqslant 2$ in any bodily region) and 15 showed both types of movements. The sample also included two patients who had a presentation dominated by multiple tics consistent with tardive Tourette syndrome; however, both these patients also showed typical dyskinetic movements in more than one body area rated at $\geqslant 2$. As shown in Table 1 , the patients showed on average moderate degrees of motor disorder, although with a wide range (mean Simpson rating scale total score 10.13 (s.d.=7.92), range 3-48). A breakdown of the scores in the tardive dyskinesia group is shown in Fig. 1.

\section{VBM comparison of the whole group of patients and controls}

As this analysis was not a principal objective of the study, the results are only briefly summarised here (see online supplementary material and Fig. DS1 for a more detailed report). There were three large clusters where the patients showed significantly reduced grey matter volume compared with the controls. One of these covered an extensive area of the medial and inferior prefrontal cortex bilaterally, reaching the dorsolateral prefrontal cortex and the pre- and postcentral gyri. This cluster also extended to the bilateral insula, parts of the temporal cortex and the right angular and right inferior and superior parietal cortex. The second cluster was seen bilaterally in the cerebellum. The third was in the middle cingulate gyrus and the supplementary motor area. The patients showed two clusters of significantly greater volume than the controls, in the cerebellar crus and the brain stem.

\section{VBM comparison of patients with and without tardive dyskinesia}

This comparison revealed a pattern of largely subcortical volume reduction in the patients with tardive dyskinesia (Fig. 2; a more detailed mapping is shown in online Fig. DS2). Two clusters affected the basal ganglia, particularly the caudate nuclei bilaterally (left side: 1200 voxels, peak at MNI $(-16,16,4)$, z-score 4.01; right side: 641 voxels, peak at MNI $(14,12,2)$ $\mathrm{z}$-score 4.16).The left-sided cluster additionally extended to both 


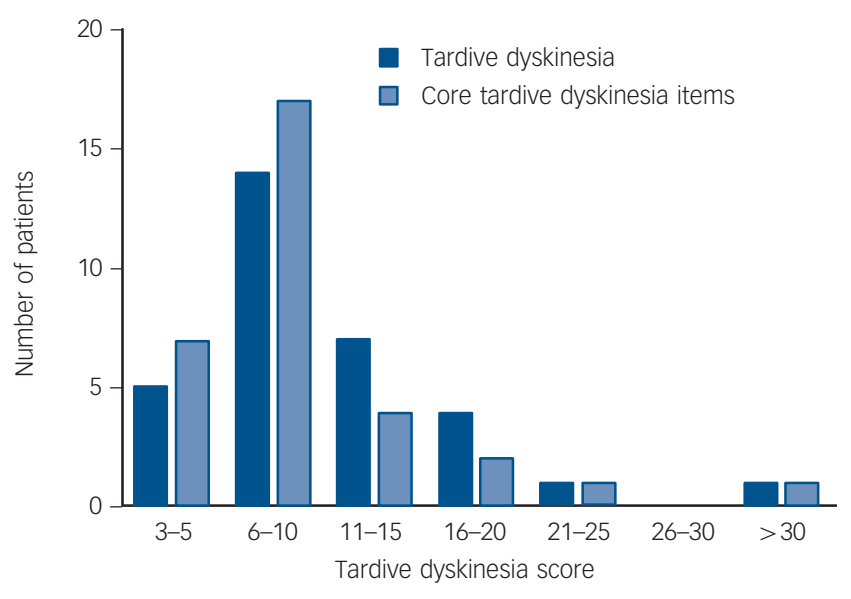

Fig. 1 Breakdown of scores on the Simpson Scale in patients with tardive dyskinesia.

thalami, and the right-sided cluster extended to the right parahippocampus, temporal pole and inferofrontal cortex. A third cluster of volume reduction affected the left parahippocampus, the amygdala, the left temporal pole and marginally the left inferior frontal cortex (323 voxels, peak at MNI $(-18,8,-22), z$-score 3.57). A fourth cluster was seen in the right cerebellum (546 voxels, peak at MNI $(14,-68,-32), z$-score 3.38$)$. There were no clusters where the patients with tardive dyskinesia showed a significantly greater volume than those without tardive dyskinesia.

Figure 3 shows the localisation of the volume reductions within the basal ganglia and thalamus. It can be seen that the caudate nucleus was most affected, the putamen to a lesser extent, and the globus pallidus only marginally. In both thalami the area affected was located predominantly medially.

\section{Volume changes in the patients with and without tardive dyskinesia compared with the controls}

To determine to what extent the regions of brain volume difference identified between patients with and without tardive dyskinesia were different from the corresponding regions in the controls we created a mask that covered all the voxels where significant differences were found between the two patient groups in the VBM comparison. This mask was then used to generate a region of interest (ROI) for grey matter volume in both patient groups and the healthy controls. The findings are shown in Figure 4(a). The controls had larger grey matter volumes than both the patients with and without tardive dyskinesia (controls: $8336 \mathrm{~mm}^{3}$ (s.d.=706); patients without tardive dyskinesia $7736 \mathrm{~mm}^{3}$ (s.d. $=734$ ); patients with tardive dyskinesia $7144 \mathrm{~mm}^{3}$ (s.d.=733)). The differences between the controls and the patients without tardive dyskinesia were significant $(t=4.4$, $P=3.0 \times 10^{-5}$, effect size: 0.8 ), as were the differences between the controls and the patients with tardive dyskinesia $(t=7.3$, $P=1.2 \times 10^{-10}$, effect size: 1.6). (Significance values for the difference between the patients with and without tardive dyskinesia in this region were not calculated, since this area had already been identified as showing significant differences in the VBM analysis.)

We also investigated volume differences between the patients and controls within the basal ganglia. To do this, an additional mask was created based solely on the basal ganglia components of the original mask (i.e. areas of caudate + putamen + globus pallidus contained in the previous mask for differences between patients with and without tardive dyskinesia). These components were identified using the standard atlases provided by the FSL software. ${ }^{28}$ Within this new mask, the patients without tardive dyskinesia showed similar but slightly larger volumes compared with the controls, but there continued to be a difference between the controls and the patients with tardive dyskinesia (controls: $2880 \mathrm{~mm}^{3}$ (s.d.=350); patients without tardive dyskinesia: $2920 \mathrm{~mm}^{3}$ (s.d. $=442$ ); patients with tardive dyskinesia $2704 \mathrm{~mm}^{3}$ (s.d.=290); Fig. 4(b)). The difference between the controls and the patients without tardive dyskinesia was not significant ( $t=0.6, P=0.6$, effect size: -0.1 ) but that between the controls and the patients with tardive dyskinesia was significant $(t=2.3$, $P=0.03$, effect size: 0.5 ).

\section{Discussion}

\section{Main findings}

Using VBM, this study found that compared with people with schizophrenia but without tardive dyskinesia, those with tardive dyskinesia showed reductions in grey matter volume that were predominantly subcortical in distribution and affected particularly the basal ganglia. Within the basal ganglia the changes were localised primarily to the caudate nucleus.

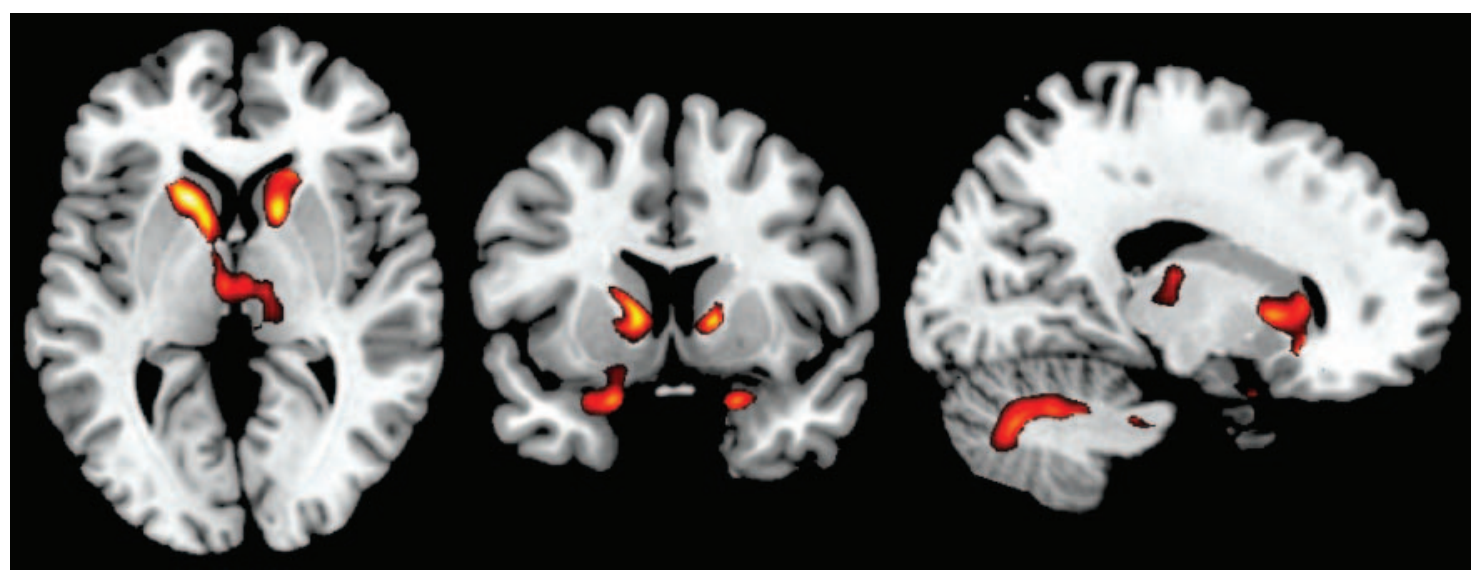

Fig. 2 Voxel-based morphometry findings.

Regions showing significant volume reduction between patients with and without tardive dyskinesia. Threshold set at $P=0.05$ corrected for multiple comparisons across space. 


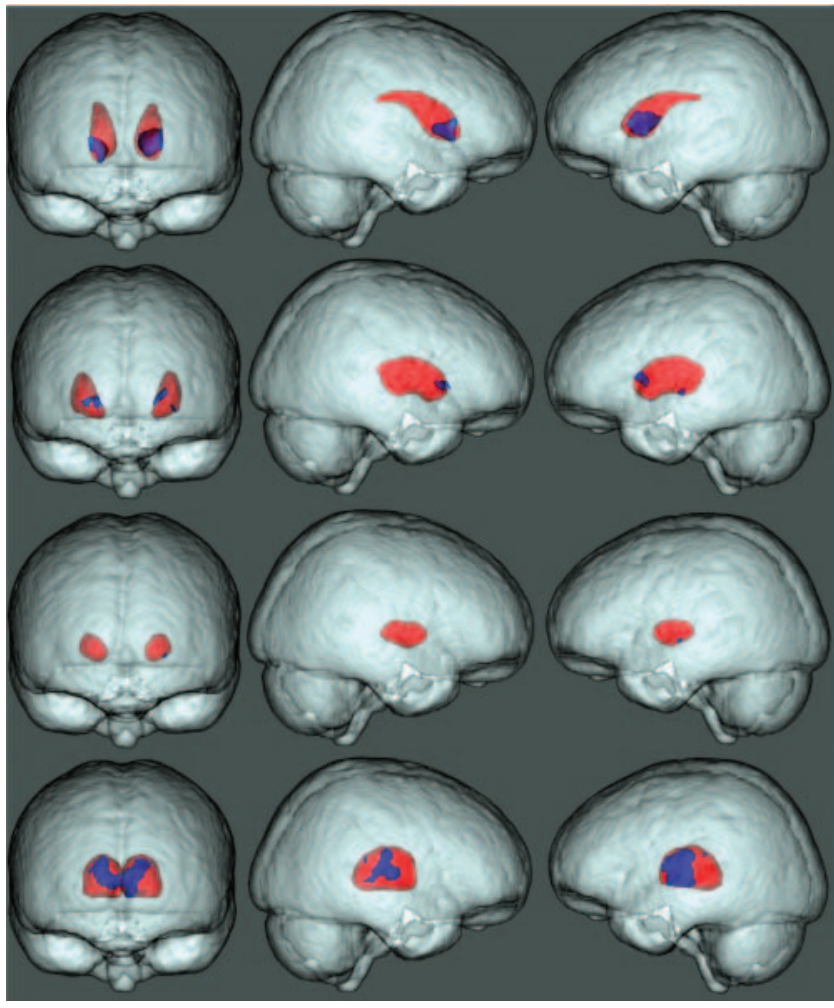

Fig. 3 Three-dimensional view showing the spatial localisation of volume reductions in the basal ganglia and thalamus.

Basal ganglia structures are depicted in red (top panel: caudate nucleus; second panel: putamen; third panel: pallidum; fourth panel: thalamus); regions showing volume reduction within these structures are shown in blue.

Our findings thus support the CT study of Bartels \& Themelis ${ }^{30}$ and the MRI study of Mion et al, ${ }^{16}$ which also found evidence of caudate nucleus changes, although as noted in the introduction other studies using conventional brain volumetry have had negative findings. To our knowledge there have been no other voxel-based studies of brain or grey matter volume in patients with and without tardive dyskinesia. However, Bai et al ${ }^{31}$ examined white matter integrity in 40 people with schizophrenia, 20 with tardive dyskinesia and 20 without, using diffusion tensor imaging (DTI) and voxel-based analysis. They found clusters of decreased fractional anisotropy in two areas that were adjacent to the basal ganglia, the white matter of the left inferior frontal gyrus and temporal lobe, although changes were also seen in other regions.

\section{Significance of findings}

A finding of brain abnormality involving the basal ganglia is what would be predicted on the basis that tardive dyskinesia is an involuntary movement disorder of the extrapyramidal type. However, the extent to which the volume differences affected the caudate nucleus more than the putamen might be considered surprising. Following the work of Alexander et $a l^{32}$ it is widely accepted that the basal ganglia are traversed by a series of cortical-subcortical-cortical 'loops', which carry both motor and non-motor information. Although these do not subdivide strictly according to nucleus, ${ }^{33}$ it is clear that the motor loop passes predominantly through the putamen. Circuits passing through the caudate nucleus are considered to be involved in higher-level control of behaviour, and lesions here tend to reproduce the apathy, perseveration, executive impairment and emotional changes seen after frontal lobe lesions. ${ }^{32,34}$ Nevertheless, it can be noted that the caudate nucleus has been found to be heavily affected in choreiform disorders such as Huntington's disease. ${ }^{35}$ It may also be relevant that the movements in tardive dyskinesia differ from those seen in other involuntary movement disorders, being more complex, rhythmical and repetitive, particularly when they affect the orofacial region; tics and stereotyped movements are also recognised features of the syndrome. ${ }^{1}$

We also found that the patients with tardive dyskinesia showed volume reductions in the thalamus. Here, the changes were located medially and centrally and therefore almost certainly involved the mediodorsal nucleus. Along with the ventroanterior/ ventrolateral (VA-VL) complex, this nucleus forms the final, thalamic relay in the cortical-basal ganglia-cortical loop systems described above. Possibly in keeping with our findings in the basal ganglia, the mediodorsal nucleus receives part of its input from circuits passing through the caudate nucleus, whereas the motor loop passes through the putamen to project to the VA-VL complex, predominantly or exclusively. ${ }^{32}$

Analysis of an ROI encompassing the areas where there were differences between the patients with and without tardive (a)

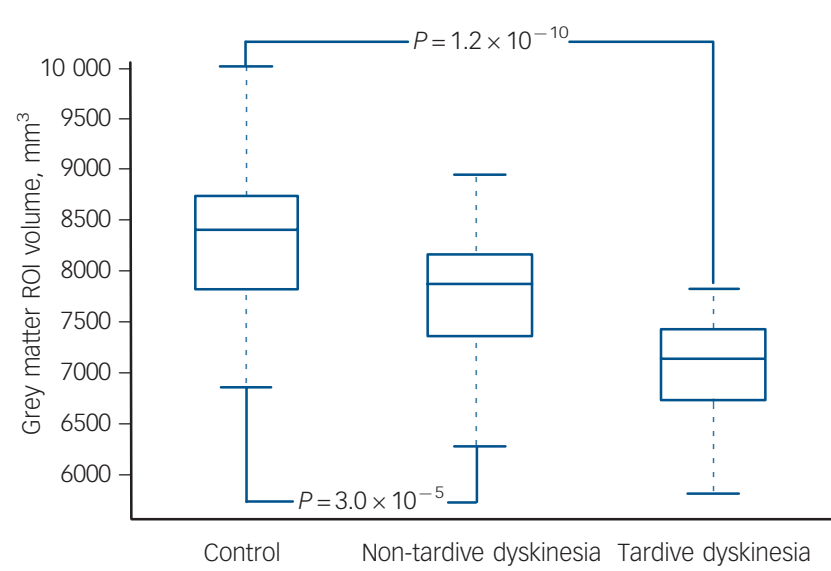

(b)

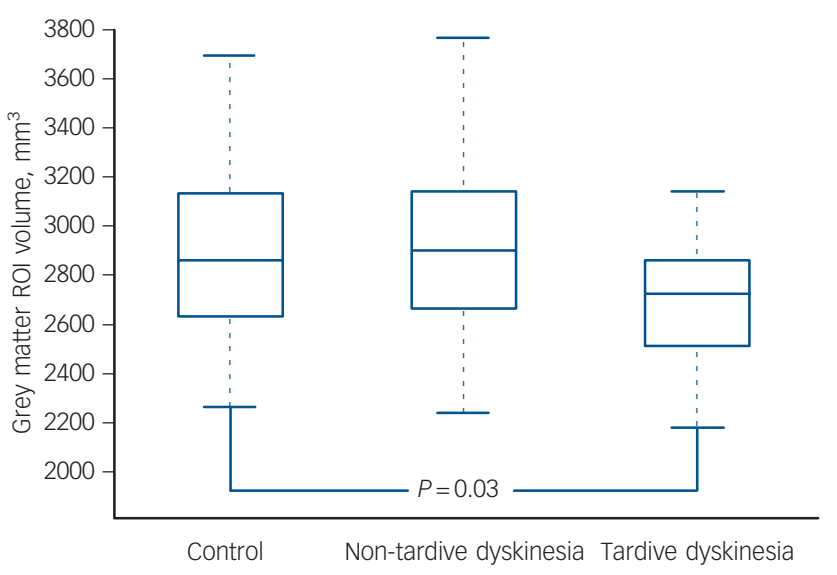

Fig. 4 Box plots of grey matter volumes in a region of interest (ROI) based on areas where the patients with tardive dyskinesia showed significant differences from those without tardive dyskinesia in the voxel-based morphometry analysis.

(a) Using a mask based on all regions where there were significant differences; (b) using a mask based solely on the basal ganglia components of the ROI. 
dyskinesia found that these regions were smaller in both of the groups of participants than in healthy controls. This suggests that tardive dyskinesia is associated with brain structural changes over and above those occurring in patients with schizophrenia who do not show tardive dyskinesia. However, this pattern did not hold true in the basal ganglia. Here, there was no significant volume difference between the controls and the patients without tardive dyskinesia, although a significant difference between controls and the patients with tardive dyskinesia was still evident. One interpretation of this latter finding is that it reflects a basal ganglia volume increase due to antipsychotic drug treatment. That such treatment-related increases occur is reasonably well-supported by the literature. ${ }^{20}$ However, this interpretation also implies that there should be basal ganglia volume reduction in patients with schizophrenia that have not been treated, which has been less consistently found, at least in the small number of studies that have used conventional MRI volume measurement (see references in Chua et $a l^{36}$ ). Recently, however, Leung et al ${ }^{37}$ carried out a meta-analysis of six VBM studies of people with first-episode schizophrenia that had never been treated and found evidence for lower grey matter volume in the caudate nucleus bilaterally, as well as in a range of frontal and temporal cortical regions. The volume reductions in the striatum and some other areas were also found to be significantly more extensive than those found in nine studies carried out on treated patients with first-episode schizophrenia.

The patients in our study showed the typical presentation of tardive dyskinesia, i.e. they were chronically ill and in most cases had been on treatment with antipsychotics for several years. It would be interesting to know whether people with schizophrenia with spontaneous dyskinesia also showed brain structural changes. Such patients are not particularly easy to find and so far only two studies have been carried out. McCreadie $e t$ a $l^{38}$ studied a sample of 62 people with chronic schizophrenia living in rural India who had never received antipsychotic treatment. Twenty-eight were found to have spontaneous dyskinesia. These patients showed no significant differences in caudate nucleus or lentiform nucleus (putamen and pallidum) volumes on MRI compared with 30 matched patients without dyskinesia. The caudate nucleus was non-significantly smaller in both patient groups than in 31 healthy controls, although the lentiform nucleus was larger (significantly on the left). Mittal et $a l^{39}$ rated involuntary movements in 30 mostly untreated patients with a diagnosis of prodromal syndrome (moderate levels of attenuated positive symptoms and/or decline in functioning in the presence of schizotypal personality disorder and/or family history of schizophrenia). They found a significant negative correlation between dyskinesia score and putamen volume, but there was no correlation with caudate nucleus volume. The results were unchanged when six participants who had received some antipsychotic treatment were excluded.

\section{Implications for aetiology}

Does a finding that tardive dyskinesia is associated with brain structural alterations have implications for the issue raised at the beginning of this article, of whether tardive dyskinesia is due to drug treatment, disease process or an interaction between the two? The answer has to be that our findings are essentially neutral on this point. One reason for this is that the study was cross-sectional in nature. Thus, for example, we cannot exclude the possibility that participants with schizophrenia who had smaller basal ganglia (and perhaps other brain structures) before they become ill are at greater risk of developing tardive dyskinesia. At first sight, our finding that tardive dyskinesia-associated volume reductions affected the caudate nucleus and putamen, which receive the bulk of the dopaminergic innervation of the basal ganglia, ${ }^{40}$ but spared the globus pallidus, might be considered to implicate antipsychotic treatment, since these drugs work by blocking dopamine receptors. However, this finding is equally consistent with a factor related to the disease process of schizophrenia, given the putative role of dopamine in this.

In conclusion, this study suggests that tardive dyskinesia is the manifestation of a process or processes that involve brain structural change, and is not just a function of neurochemical changes, for example in postsynaptic $\mathrm{D}_{2}$ receptor numbers, as previously hypothesised. At the same time, this finding should not, by itself, be taken to imply that antipsychotic drugs can cause brain volume reductions, as has recently been claimed in animals ${ }^{41}$ and patients with schizophrenia. ${ }^{42}$

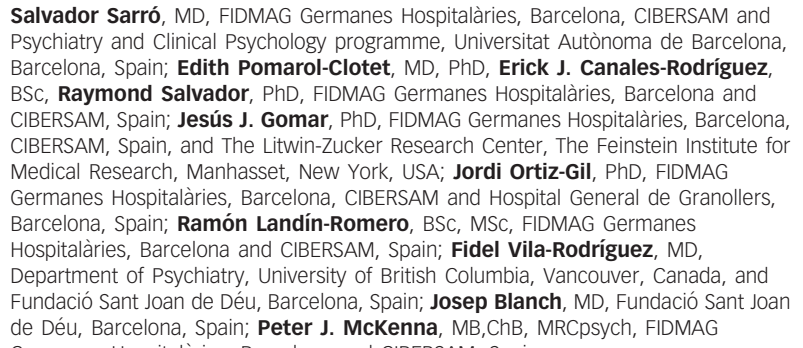

Salvador Sarró, MD, FIDMAG Germanes Hospitalàries, Barcelona, CIBERSAM and Psychiatry and Clinical Psychology programme, Universitat Autònoma de Barcelona, Barcelona, Spain; Edith Pomarol-Clotet, MD, PhD, Erick J. Canales-Rodríguez, Barcelona, Spain; Edith Pomarol-Clotet, MD, PhD, Erick J. Canales-Rodriguez,
BSC, Raymond Salvador, PhD, FIDMAG Germanes Hospitalàries, Barcelona and CIBERSAM, Spain; Jesús J. Gomar, PhD, FIDMAG Germanes Hospitalàries, Barcelona, CIBERSAM, Spain, and The Litwin-Zucker Research Center, The Feinstein Institute for Medical Research, Manhasset, New York, USA; Jordi Ortiz-Gil, PhD, FIDMAG Germanes Hospitalàries, Barcelona, CIBERSAM and Hospital General de Granollers, Barcelona, Spain; Ramón Landín-Romero, BSC, MSc, FIDMAG Germanes Barcelona, Spain; Ramón Landín-Romero, BSC, MSC,
Hospitalies, Barcelona and CIBERSAM, Spain; Fidel Vila-Rodríguez, MD, Hospitalaries, Barcelona and CIBERSAM, Spain; Fidel Vila-Rodriguez, MD,
Department of Psychiatry, University of British Columbia, Vancouver, Canada, and Fundació Sant Joan de Déu, Barcelona, Spain; Josep Blanch, MD, Fundació Sant Joan de Déu, Barcelona, Spain; Peter J. McKenna, MB,ChB, MRCpsych, FIDMAG Germanes Hospitalàries, Barcelona and CIBERSAM, Spain

Correspondence: Peter McKenna, FIDMAG Germanes Hospitalàries, C.I Dr Antoni Pujadas 38, 08830 - Sant Boi de Llobregat, Barcelona, Spain. Email: pmckenna@fidmag.com

First received 8 May 2012, final revision 26 Jun 2012, accepted 24 Oct 2012

\section{Funding}

This work was supported by (a) a Marie Curie Reintegration Grant (MERG-CT-2004-511069 given to E.P.-C.); (b) the Centro de Investigación Biomédica en Red de Salud Mental (CIBERSAM): (c) several grants from the Instituto de Salud Carlos III (Miguel Servet Research Contract to R.S. (CP07/00048) and to E.P.-C. (CP10/00596); Intensification grant to S.S. (10/ 231); Research Project to E.P.-C. (PI05/2693)) and (d) the Comissionat per a Universitats i Recerca del DIUE from the Catalonian Government (2009SGR211).

\section{References}

1 Cunningham Owens DG. A Guide to the Extrapyramidal Side-Effects of Neuroleptic Drugs. Cambridge University Press, 1999

2 Fenton WS. Prevalence of spontaneous dyskinesia in schizophrenia. J Clin Psychiatry 2000; 61 (suppl 4): 10-4.

3 Owens DG, Johnstone EC, Frith CD. Spontaneous involuntary disorders of movement: their prevalence, severity, and distribution in chronic schizophrenics with and without treatment with neuroleptics. Arch Gen Psychiatry 1982; 39: 452-61.

4 Mccreadie RG, Thara R, Kamath S, Padmavathy R, Latha S, Mathrubootham $\mathrm{N}$, et al. Abnormal movements in never-medicated Indian patients with schizophrenia. Br J Psychiatry 1996; 168: 221-6.

5 Whitty PF, Owoeye O, Waddington JL. Neurological signs and involuntary movements in schizophrenia: intrinsic to and informative on systems pathobiology. Schizophr Bull 2009; 35: 415-24.

6 Crow TJ, Owens DG, Johnstone EC, Cross AJ, Owen F. Does tardive dyskinesia exist? Mod Probl Pharmacopsychiatry 1983; 21: 206-19.

7 Casey DE. Tardive dyskinesia: pathophysiology and animal models. J Clin Psychiatry 2000; 61 (suppl 4): 5-9.

8 Klawans HL, Weiner WJ. The pharmacology of choreatic movement disorders. Prog Neurobiol 1976; 6: 49-80.

9 Gerlach J. Tardive dyskinesia. Dan Med Bull 1979; 26: 209-45.

10 Mackay AV, Iversen LL, Rossor M, Spokes E, Bird E, Arregui A, et al. Increased brain dopamine and dopamine receptors in schizophrenia. Arch Gen Psychiatry 1982; 39: 991-7.

11 Cross AJ, Crow TJ, Ferrier IN, Johnson JA, Johnstone EC, Owen F, et al. Chemical and structural changes in the brain in patients with movement disorder. Psychopharmacology Suppl 1985; 2: 104-10. 
12 Kornhuber J, Riederer P, Reynolds GP, Beckmann H, Jellinger K, Gabriel E. $3 \mathrm{H}$-spiperone binding sites in post-mortem brains from schizophrenic patients: relationship to neuroleptic drug treatment, abnormal movements, and positive symptoms. J Neural Transm 1989; 75: 1-10.

13 Adler CM, Malhotra AK, Elman I, Pickar D, Breier A. Amphetamine-induced dopamine release and post-synaptic specific binding in patients with mild tardive dyskinesia. Neuropsychopharmacology 2002; 26: 295-300.

14 Owens DG, Johnstone EC, Crow TJ, Frith CD, Jagoe JR, Kreel L. Lateral ventricular size in schizophrenia: relationship to the disease process and its clinical manifestations. Psychol Med 1985; 15: 27-41.

15 Hoffman WF, Casey DE. Computed tomographic evaluation of patients with tardive dyskinesia. Schizophr Res 1991; 5: 1-12.

16 Mion CC, Andreasen NC, Arndt S, Swayze 2nd VW, Cohen GA. MRI abnormalities in tardive dyskinesia. Psychiatry Res 1991; 40: 157-66.

17 Elkashef AM, Buchanan RW, Gellad F, Munson RC, Breier A. Basal ganglia pathology in schizophrenia and tardive dyskinesia: an MRI quantitative study. Am J Psychiatry 1994; 151: 752-5.

18 Waddington JL, O'Callaghan E, Buckley P, Madigan C, Redmond O, Stack JP, et al. Tardive dyskinesia in schizophrenia. Relationship to minor physical anomalies, frontal lobe dysfunction and cerebral structure on magnetic resonance imaging. Br J Psychiatry 1995; 167: 41-4.

19 Wright IC, Rabe-Hesketh S, Woodruff PW, David AS, Murray RM, Bullmore ET. Meta-analysis of regional brain volumes in schizophrenia. Am J Psychiatry 2000; 157: 16-25.

20 Brandt GN, Bonelli RM. Structural neuroimaging of the basal ganglia in schizophrenic patients: a review. Wien Med Wochenschr 2008; 158: 84-90.

21 American Psychiatric Association. Diagnostic and Statistical Manual of Mental Disorders (4th edn) (DSM-IV). APA, 1994.

22 Simpson GM, Lee JH, Zoubok B, Gardos G. A rating scale for tardive dyskinesia. Psychopharmacology (Berl) 1979; 64: 171-9.

23 Schooler NR, Kane JM. Research diagnoses for tardive dyskinesia. Arch Gen Psychiatry 1982; 39: 486-7.

24 Peralta V, Cuesta MJ. Psychometric properties of the positive and negative syndrome scale (PANSS) in schizophrenia. Psychiatry Res 1994; 53: 31-40.

25 Del Ser T, Gonzalez-Montalvo Jl, Martinez-Espinosa S, Delgado-Villapalos C, Bermejo F. Estimation of premorbid intelligence in Spanish people with the Word Accentuation Test and its application to the diagnosis of dementia. Brain Cogn 1997; 33: 343-56.

26 Wechsler D. Wechsler Adult Intelligence Scale (3rd edn). The Psychological Corporation, 1997.

27 Good CD, Johnsrude IS, Ashburner J, Henson RN, Friston KJ, Frackowiak RS A voxel-based morphometric study of ageing in 465 normal adult human brains. Neuroimage 2001; 14: 21-36.

28 Smith SM, Jenkinson M, Woolrich MW, Beckmann CF, Behrens TE, Johansen-Berg $\mathrm{H}$, et al. Advances in functional and structural MR image analysis and implementation as FSL. Neuroimage 2004; 23 (suppl 1): S208-19.

29 Smith SM. Fast robust automated brain extraction. Hum Brain Mapp 2002; 17: $143-55$.

30 Bartels $\mathrm{M}$, Themelis J. Computerized tomography in tardive dyskinesia. Evidence of structural abnormalities in the basal ganglia system. Arch Psychiatr Nervenkr 1983; 233: 371-9.

31 Bai YM, Chou KH, Lin CP, Chen IY, Li CT, Yang KC, et al. White matter abnormalities in schizophrenia patients with tardive dyskinesia: a diffusion tensor image study. Schizophr Res 2009; 109: 167-81.

32 Alexander GE, DeLong MR, Strick PL. Parallel organization of functionally segregated circuits linking basal ganglia and cortex. Annu Rev Neurosci 1986; 9: $357-81$.

33 Parent A, Hazrati LN. Functional anatomy of the basal ganglia. I. The cortico-basal ganglia-thalamo-cortical loop. Brain Res Brain Res Rev 1995; 20: 91-127.

34 cummings JL. Anatomic and behavioral aspects of frontal-subcortical circuits. Ann N Y Acad Sci 1995; 769: 1-13.

35 Quinn N, Schrag A. Huntington's disease and other choreas. J Neurol 1998; 245: 709-16.

36 Chua SE, Cheung C, Cheung V, Tsang JT, Chen EY, Wong JC, et al. Cerebral grey, white matter and csf in never-medicated, first-episode schizophrenia. Schizophr Res 2007; 89: 12-21.

37 Leung M, Cheung C, Yu K, Yip B, Sham P, Li Q, et al. Gray matter in firstepisode schizophrenia before and after antipsychotic drug treatment. Anatomical likelihood estimation meta-analyses with sample size weighting. Schizophr Bull 2011; 37: 199-211.

38 McCreadie RG, Thara R, Padmavati R, Srinivasan TN, Jaipurkar SD. Structural brain differences between never-treated patients with schizophrenia, with and without dyskinesia, and normal control subjects: a magnetic resonance imaging study. Arch Gen Psychiatry 2002; 59: 332-6.

39 Mittal VA, Daley M, Shiode MF, Bearden CE, O'Neill J, Cannon TD. Striatal volumes and dyskinetic movements in youth at high-risk for psychosis. Schizophr Res 2010; 123: 68-70.

40 Bjorklund A, Dunnett SB. Dopamine neuron systems in the brain: an update. Trends Neurosci 2007; 30: 194-202.

41 Dorph-Petersen KA, Pierri JN, Perel JM, Sun Z, Sampson AR, Lewis DA. The influence of chronic exposure to antipsychotic medications on brain size before and after tissue fixation: a comparison of haloperidol and olanzapine in macaque monkeys. Neuropsychopharmacology 2005; 30: 1649-61.

42 Ho BC, Andreasen NC, Ziebell S, Pierson R, Magnotta V. Long-term antipsychotic treatment and brain volumes: a longitudinal study of first-episode schizophrenia. Arch Gen Psychiatry 2011; 68: 128-37. 